\title{
RAPID TESTING: ANALYSIS OF GNSS RAPID STATIC OBSERVATIONS SUITABILITY FOR ENGINEERING SURVEYS IN URBAN ENVIRONMENTS IN THE TIME OF COVID-19
}

\author{
Serafin Farley M. Meneses III \\ Department of Geodetic Engineering, University of the Philippines, Diliman, Quezon City - smmeneses@ up.edu.ph
}

KEY WORDS: GNSS, GPS, GLONASS, BeiDou, Engineering Surveys, Land Surveying

\begin{abstract}
:
This study aims to determine which rapid static observation durations would have acceptable accuracy for engineering surveys in urban environments (i.e. Metro Manila) in the time of COVID-19. Due to health concerns caused by the COVID-19 pandemic, Metro Manila has experienced various restrictions in mobility and time spent in public spaces in recent months. This has affected not only the lives and ways of work of the so-called front liners like nurses, doctors, and primary health care workers, but also the public at large which includes Land Surveyors. It is for this reason that this study was conducted, since a balance must be struck between the aim to get accurate engineering survey results and the health and safety of those who are conducting the measurements. Hence, the shortest possible time to conduct rapid static GNSS observation durations with acceptable results must be determined while ensuring that the conduct of the field survey would still be in compliance to the minimum health protocols (i.e. no mass gathering, maintenance of physical distancing, short time of interaction, etc.) set by the national government.
\end{abstract}

For this study, rapid static observations were made at varying time intervals (i.e. 2 minutes, 5 minutes, 10 minutes, 15 minutes, 30 minuntes, 1 hour, and 2 hours) at locations (i.e. open, with minimal obstructions, with significant obstructions) that would simulate archetypal situations when conducting engineering surveys in urban environments. Results were computed using fully constrained least square adjustment and results show that if all GNSS satellites are used in the computations, all time intervals would yield acceptable RMSE values, both for the horizontal $(5 \mathrm{~mm}$ to $2 \mathrm{~cm})$ and vertical $(1 \mathrm{~cm}$ to $4 \mathrm{~cm})$, for engineering surveys. However, if not all GNSS signals are available, it is best to use at least two GNSS satellite constellations (i.e. GPS-BeiDou, GPS-Glonass, Glonass-BeiDou) so that rapid static observations with acceptable accuracy can be made for as short as 5 minutes. For the "classical" accuracy standards, all rapid static observation durations yielded Order B relative precisions for the horizontal while most, except for the 30-minute duration, which yielded Third Order level results for the vertical.

\section{INTRODUCTION}

The COVID-19 pandemic has caused numerous disruptions in the way people do things (i.e. the "new normal"), especially in the conduct of their work (i.e. shift from face-to-face interactions to work-from-home arrangements and/or skeleton workforce, etc.). Land Surveyors are not exempt from such changes and challenges (RICS, 2020) and the pandemic has certainly impacted the way Surveyors in Manila conduct Global Navigation Satellite Systems (GNSS) observations for land and engineering surveys. COVID-19 induced restrictions, like minimum physical distancing and travel curfews, have introduced additional burdens to Land Surveyors in doing GNSS observations in urban environments, where there are already a lot of possible sources of errors like multi-path sources and signal disruptions especially when observations are made in urban canyons, narrow pathways, and densely populated areas.

Given the current situation and due to the natural desire of Land Surveyors to abide to the strictest professional standards, it became imperative to find ways to adapt and improve ways to conduct work, particularly in doing engineering surveys in urban environments using GNSS technology. It is recognized that a balance must be struct between accuracy and precision of Geodetic observations vis-a-vis health and safety of the field personnel doing the observations. It is for this reason that this academic study on rapid static GNSS observations was initiated.
For this study, rapid GNSS observations were made at three points that simulates typical urban settings in Manila. Observation durations were 2 minutes, 5 minutes, 10 minutes, 15 minutes, 30 minutes, 1 hour, and 2 hours. Typically, more than 30 minutes of observations are no longer considered as rapid, however the definition by Online Positioning User Service (OPUS) (NGS, 2020) of rapid static duration (i.e. up to 2 hours) was adapted in this research. All rapid static observations were computed using fully constrained least squares adjustment and their values were compared to reference coordinates of the occupied points which were obtained by either observing the occupied points for more than three hours or through National Mapping and Resource Information Agency (NAMRIA) Ground Control Point (GCP) certification.

Results show that all rapid static observation durations were able to meet the "classical" Order B relative precision (i.e. $1: 1,000,000)$ in the horizontal while most observation durations were near the Third Order accuracy standard in the vertical except for the 30-minute duration. Note that the generally accepted accuracy standard for engineering surveys is the Third Order accuracy (Ogaja, 2011).

For the RMSE, it was observed that all rapid static observation durations give acceptable values for engineering surveys for both the horizontal $(5 \mathrm{~mm}$ to $2 \mathrm{~cm})$ and vertical $(1 \mathrm{~cm}$ to $4 \mathrm{~cm})$ observations if all GNSS satellites are used for the computation. However, if it is not possible to use all GNSS satellites, it is best to use at least two satellite constellations (i.e. GPS-BeiDou, GPS-Glonass, or Glonass-BeiDou) in the GNSS data 
computations so that rapid static observations for engineering surveys in urban settings can be made for as short as 5 minutes only.

\section{METHODOLOGY}

\subsection{Study Area}

Given the travel restrictions imposed by the Inter-Agency Task Force for the Management of Emerging Infectious Diseases (IATF) during community quarantine periods, all observations were done around Katipunan Avenue, located in Balara, Quezon City for convenient access, shown in Figure 1 below.
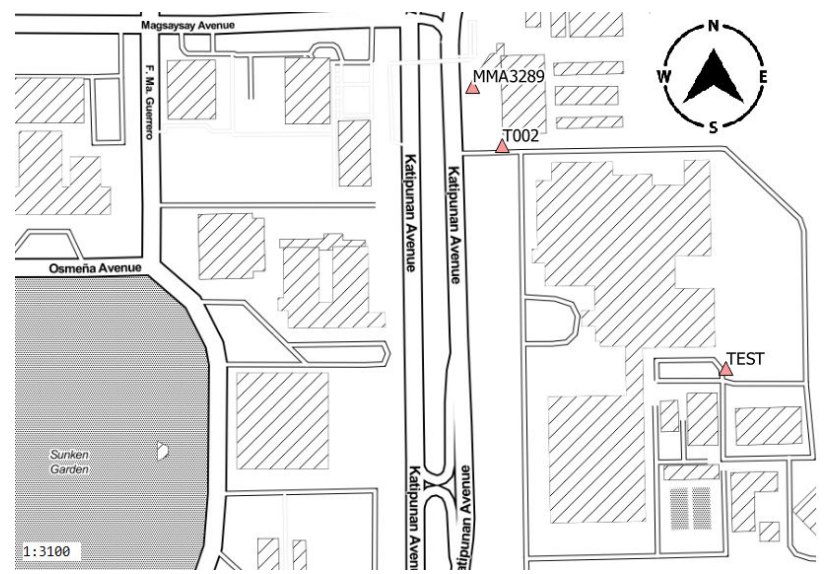

Figure 1. Location of study area, chosen because of its convenience due to its proximity to NAMRIA controls and on how it simulates actual ground conditions in doing surveying in urban settings.

The observation points were chosen in order to simulate actual field conditions when getting locations of typical points of interest, like manholes, electric poles, valves, and telephone communication boxes, in urban areas. MMA3289, which is also a fourth-order NAMRIA GCP, simulated an observation point with an almost open view of the sky (Fig. 2), T002 resembles locations with minimal obstructions (Fig. 3), while TEST corresponds to points where there are lot of possible sources of multipath error and signal interferences (Fig. 4).

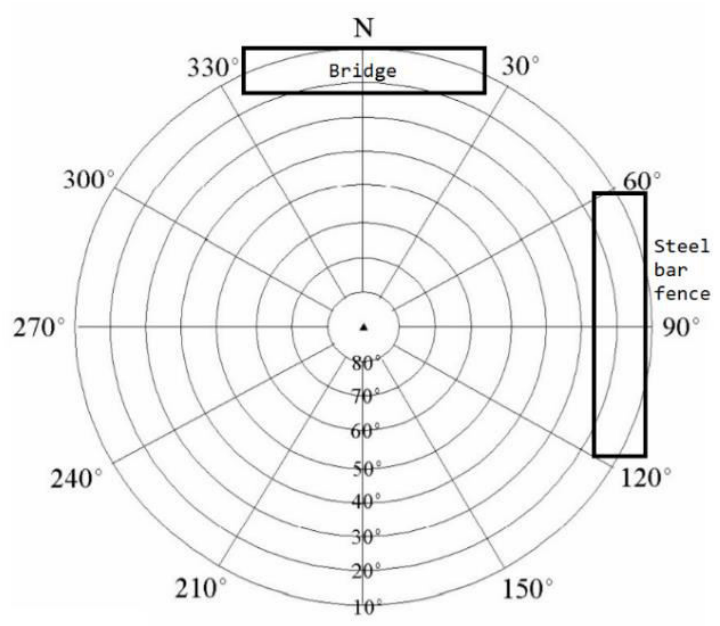

Figure 2. Visibility obstruction diagram of MMA3289, showing that the observation point has an almost very clear view of the sky.

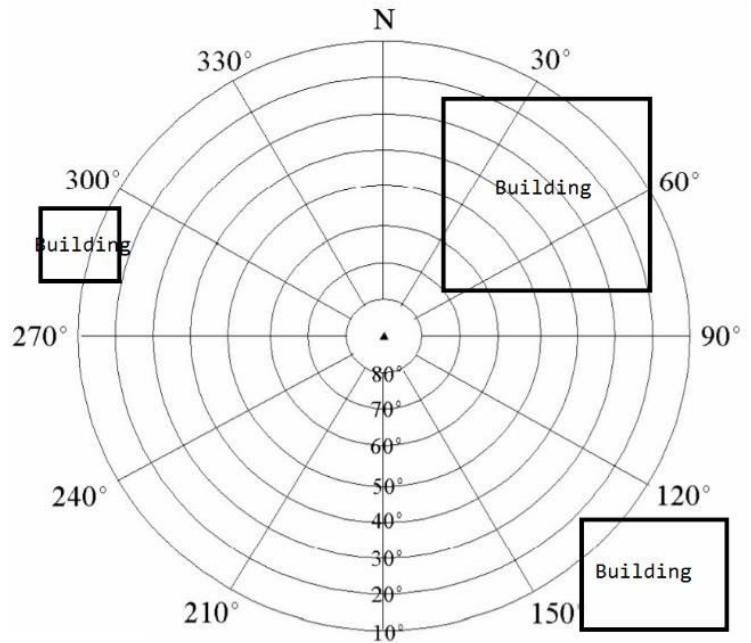

Figure 3. Visibility obstruction diagram of T002, showing minimal obstruction except on the northeast side.

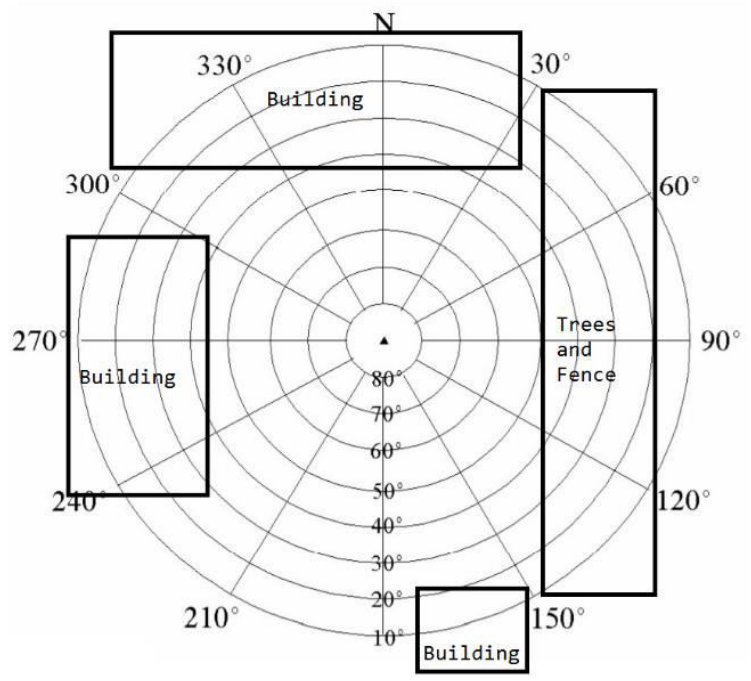

Figure 4. Visibility obstruction diagram of station TEST, simulating observations done in urban canyons and highly dense areas in Manila.

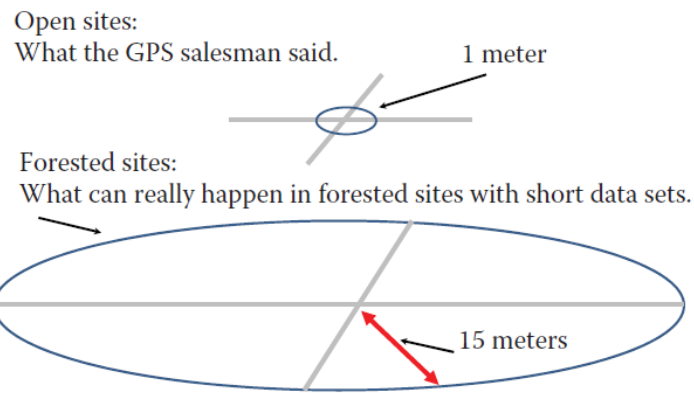

Figure 5. Manufacturer specified precision are usually for open sites and long datasets. Adapted from Ogaja (2011). 


\subsection{Equipment and Software}

GNSS observations on the simulation points were done using Spectra Precision SP80/85 antenna with Ranger 3/7 controller. Logging interval was set to 2 seconds and mask angle was set to 10 degrees, with all GNSS, namely GPS, GLONASS, BeiDou, QZSS, and Galileo, constellations enabled. Based from manufacturer's specification, the unit has a root mean square error (RMSE) of $3 \mathrm{~mm}+0.5 \mathrm{ppm}$ and $5 \mathrm{~mm}+0.5 \mathrm{ppm}$ in the horizontal and vertical observations respectively for rapid static observations.

For the reference stations, static logs of four continuously operating reference stations (CORS) were used. GNSS equipment installed in these stations are Satlab SLX1 receivers with AT35101H antenna. The design of the installation of the CORS network were based on Australia's Tier 3 CORS guidelines (ICSM, 2014). These four stations were all registered to NAMRIA and are second-order GCPs. Logging intervals of the CORS receivers were set to every second, mask angles to 15 degrees, and all were enabled to track GPS, GLONASS, GALILEO, and BeiDou satellites. Maximum observation baseline for the study is approximately $24 \mathrm{~km}$.

Based from their technical specifications, the RMSE of SLX1 for post-processed static horizontal and vertical observations are $2.5 \mathrm{~mm}+0.5 \mathrm{ppm}$ and $5 \mathrm{~mm}+0.5 \mathrm{ppm}$ respectively. Along with the declared RMSE of the Spectra Precision equipment, it is good practice to validate these declared precision values of Satlab as shown by Ogaja (2011) (Fig. 5).

For the baseline processing of GNSS data, Trimble Business Center (TBC) was used. Coordinate system was set to Universal Transverse Mercator (UTM) Zone 51 North (Z51N) for the projection and World Geodetic System 1984 (WGS 84) for the datum. Geoid was set to Earth Gravitational Model 2008 (EGM 08). Baseline processing acceptance criteria was set to the default values of $0.05 \mathrm{~m}+1 \mathrm{ppm}$ and $0.1 \mathrm{~m}+1 \mathrm{ppm}$ for the horizontal and vertical observations. Network adjustment was always performed after baseline processing, wherein a fully constrained solution was employed, using the four CORS stations as survey-grade controls for the adjustment in northings, eastings, and ellipsoidal height. Observed rapid ephemeris corrections, which are downloaded from National Aeronautics and Space Administration's (NASA) Crustal Dynamics Data Information System (CDDIS) (https://cddis.nasa.gov/) were also included in the network adjustment computations.

\subsection{On Real-time Kinematic and Rapid Static Observations considerations}

Real-time kinematic (RTK) GNSS observations are carrierphase based positioning method that uses at least two receivers tracking the same set of satellites. A "base" station receiver is established on a known point and this base receiver will transmit its GNSS measurements and coordinates to the "rover" receiver through a communication link (i.e. Network Transport of RTCM via Internet Protocol a.k.a. NTRIP or through radio) (Fig. 6). The rover will then compute its position and coordinates through on-the-fly (OTF) ambiguity resolution (ElRabbany, 2002). If conditions are optimal (i.e. there is no multipath, position dilution of precision is low, large number of satellites are tracked, etc.), the RTK method can fix ambiguity in seconds, with little degradation in positional accuracy (Van Sickle, 2001).

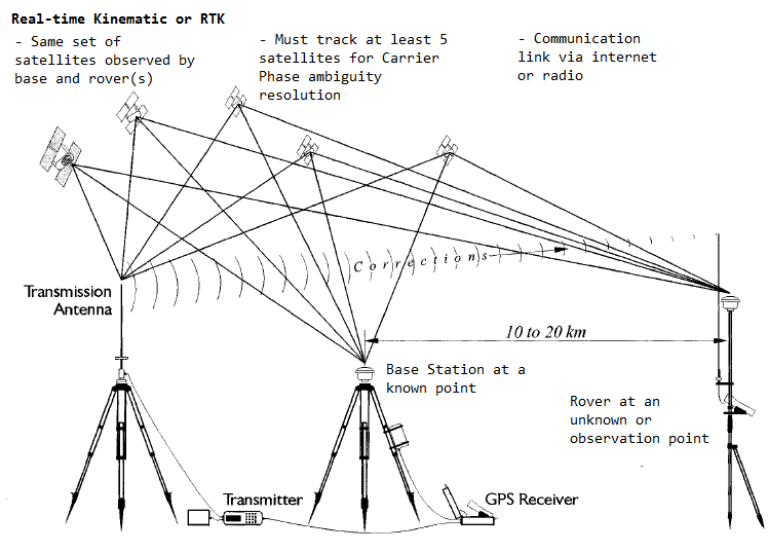

Figure 6. Diagram of typical GNSS set-up for RTK observations. Adapted from Van Sickle (2001).

GNSS rapid static observations are basically the same as static observations, only that the duration of observations is shorter. How short rapid static observations needed to be observed typically depends on the purpose of the survey, type of instrument used, location of the observation, size of the area to be surveyed, among others. However, it should be noted that COVID-19 restrictions, like minimum physical distancing and geographic lockdown protocols, should also now be considered when selecting the duration of rapid static observations. For baselines less than $20 \mathrm{~km}$ in length, Ghilani and Wolf (2012) recommends at least $10 \mathrm{~min}+1 \mathrm{~min} / \mathrm{baseline}$ length be done while Gopi, et.al. (2018) recommends at least 20 minutes of observation. However, for this academic study, the United States National Geodetic Survey (US NGS) OPUS definition of rapid static durations for shall be adopted (i.e. GNSS observations that are 2 hours long or less) (NGS, 2020).

While RTK solutions for GNSS observations are now widely available and typically offer faster observation time, the reliability and accuracy of such technique when used in urban areas, like in Metro Manila where there are radio signal obstructions, weak internet connectivity, and lots of sources of multipath error, are still limited and challenging (Fan, 2019; Li, 2018). It is for reason, in addition to the current COVID-19 situation wherein it is desired that fieldworks be done in the most efficient manner in accordance to IATF regulations (i.e. accurate results in relatively short observation times, minimal face-to-face interaction in the conduct of the field works, and reduced possibility of repeat work due to unresolved ambiguities), only the rapid static GNSS observation methodology is considered for this study and not RTK observations.

\subsection{Observations and computations}

To be able to maintain almost similar conditions during the observations, all three locations were observed simultaneously using the same type of instrument (i.e. SP80/85). The observations at each station were staggered to 2 minutes, 5 minutes, 10 minutes, 15 minutes, 30 minutes, 1 hour and 2 hours intervals with a 10-minute break between observations. The reason for choosing these observation intervals was to determine the most practical, in terms of length of observation and minimal RMSE, duration for rapid static observations.

For the computations, SATLAB SLX1's raw data were converted to RINEX 3.02 before it was loaded into TBC. SP80/85 raw data can be directly read by Trimble's processing 
software. It was also ensured that the correct metadata like height of instrument and measurement style were inputted to each of the observations. Appropriate ephemeris data are also imported from NASA CDDIS and were included in the network adjustment computations.

The NAMRIA-certified coordinates were used as reference data for the four reference stations and baselines were processed individually for each of the stations per each rapid static observation using all GNSS available (i.e. GPS + GLONASS + BeiDou + Galileo + QZSS). Once a fix has been obtained for the station after baseline processing, minimal and constrained network adjustment are then done in succession. This process is then repeated for each of the GNSS constellation individually, namely GPS only, GLONASS only, BeiDou only, to also assess the performance of individual constellations when used for rapid static observations. Galileo did not produce enough fixed solutions to merit inclusion in the analysis of results.

The coordinates obtained for each of the station per rapid static observation are then compared against established coordinates of the occupation points to get their RMSE values. For MMA3289, the official NAMRIA coordinates was used as reference values while for T002 and TEST, values from previous GNSS campaigns (with more than 3 hours of observation) were used.

\section{RESULTS}

\subsection{Rapid static observations}

Tables 1, 2, and 3 below shows the horizontal and vertical deviations (i.e. residuals) of the observations from the reference coordinates per rapid static observation duration at each of the stations using all observed GNSS signals.

\begin{tabular}{|l|c|c|}
\hline MMA3289 & Horizontal $(\mathrm{m})$ & Vertical $(\mathrm{m})$ \\
\hline $2 \mathrm{~min}$ & 0.125 & -0.805 \\
\hline $5 \mathrm{~min}$ & 0.123 & -0.823 \\
\hline $10 \mathrm{~min}$ & 0.113 & -0.816 \\
\hline $15 \mathrm{~min}$ & 0.107 & -0.796 \\
\hline $30 \mathrm{~min}$ & 0.107 & -0.805 \\
\hline 1 hour & 0.113 & -0.817 \\
\hline 2 hours & 0.108 & -0.825 \\
\hline
\end{tabular}

Table 1. Deviations in horizontal and vertical for MMA3289. It can be seen that there is a constant offset of about $0.114 \mathrm{~m}$ and $0.812 \mathrm{~m}$ for the horizontal and vertical deviations respectively.

These biases are removed in all analysis henceforth.

\begin{tabular}{|l|c|c|}
\hline TEST & Horizontal $(\mathrm{m})$ & Vertical $(\mathrm{m})$ \\
\hline $2 \mathrm{~min}$ & 0.033 & -0.031 \\
\hline $5 \mathrm{~min}$ & 0.017 & 0.017 \\
\hline $10 \mathrm{~min}$ & 0.022 & -0.011 \\
\hline $15 \mathrm{~min}$ & 0.025 & -0.016 \\
\hline $30 \mathrm{~min}$ & 0.002 & -0.060 \\
\hline 1 hour & 0.013 & -0.020 \\
\hline 2 hours & 0.009 & -0.013 \\
\hline
\end{tabular}

Table 2. Deviations in horizontal and vertical for TEST.

\begin{tabular}{|l|c|c|}
\hline T002 & Horizontal $(\mathrm{m})$ & Vertical $(\mathrm{m})$ \\
\hline $2 \mathrm{~min}$ & 0.008 & -0.001 \\
\hline
\end{tabular}

\begin{tabular}{|l|c|c|}
$5 \mathrm{~min}$ & 0.020 & 0.001 \\
\hline $10 \mathrm{~min}$ & 0.015 & -0.013 \\
\hline $15 \mathrm{~min}$ & 0.008 & 0.018 \\
\hline $30 \mathrm{~min}$ & 0.022 & -0.022 \\
\hline 1 hour & 0.015 & -0.003 \\
\hline 2 hours & 0.004 & -0.003 \\
\hline
\end{tabular}

Table 3. Deviations in horizontal and vertical for T002.

\begin{tabular}{|l|c|c|}
\hline $\begin{array}{l}\text { MMA3289 } \\
\text { w/o biases }\end{array}$ & Horizontal $(\mathrm{m})$ & Vertical (m) \\
\hline 2min & 0.013 & 0.007 \\
\hline $5 \mathrm{~min}$ & 0.013 & -0.011 \\
\hline $10 \mathrm{~min}$ & 0.012 & -0.004 \\
\hline $15 \mathrm{~min}$ & 0.007 & 0.016 \\
\hline $30 \mathrm{~min}$ & 0.008 & 0.007 \\
\hline $1 \mathrm{hr}$ & 0.003 & -0.005 \\
\hline $2 \mathrm{hr}$ & 0.008 & -0.013 \\
\hline
\end{tabular}

Table 4. Deviations in horizontal and vertical for MMA3289 without the detected shifts in position and elevation.

As can be seen on Table 1 , there is an about $0.114 \mathrm{~m}$ bias in the horizontal position and around $-0.812 \mathrm{~m}$ bias in elevation for MMA3289. MMA3289 is a fourth order NAMRIA geodetic control point with Northings and Eastings of $1621288.570 \mathrm{~m}$ and $292647.670 \mathrm{~m}$ respectively, expressed in UTM Zone 51 North projection. These "biases" are removed in all the analysis in the proceeding Sections of this study. The rest of the reference coordinates values are seen on Table 5.

\begin{tabular}{|l|c|c|c|}
\hline Point ID & Northings $(\mathrm{m})$ & Eastings $(\mathrm{m})$ & Elevation $(\mathrm{m})$ \\
\hline TEST & 1621110.062 & 292806.317 & 62.971 \\
\hline T002 & 1621251.222 & 292666.182 & 69.338 \\
\hline MMA3289 & 1621288.570 & 292647.670 & 73.104 \\
\hline
\end{tabular}

Table 5. Values of coordinates of the test points.

As can be seen in the Tables 2, 3, and 4, the absolute maximum deviation for the horizontal is around $0.033 \mathrm{~m}$ for the 2-minute observation at TEST and around $0.06 \mathrm{~m}$ for the vertical, also at TEST for the 30-minute observation. Absolute minimum deviation of around $0.003 \mathrm{~m}$ for the horizontal for the 1-hour observation at MMA3289 and $0.001 \mathrm{~m}$ at the 2- and 5-minute observations at T002 are also noted.

Based from the results shown in Tables 2, 3, and 4, average misclosures and relative precisions are computed and shown in Table 6. It can be seen that all observation durations meet the Order B for horizontal control survey accuracy standards of US Federal Geodetic Control Subcommittee (FGCC, 1984) (Fig. 7). Using the maximum observation baseline of $24 \mathrm{~km}$, for vertical accuracy, only the 30-minute observation is considerably outside the minimum Third Order classification standard (Fig. $8)$.

Note that while these "classical" accuracy standards were primarily designed to support geodetic control surveys, it is widely accepted that the Third Order classifications are suitable for engineering surveys (Ogaja, 2011), which is the target survey activity of this study. It is also noteworthy to mention that while updated accuracy standards exists, especially for GNSS observations (FGCC, 1998), local land surveying 
guidelines still refer to relative accuracy standards for evaluating land survey measurements (LMB, 2010), hence the inclusion of these standards in this study.

\begin{tabular}{|l|c|c|l|l|}
\hline \multirow{2}{*}{} & \multicolumn{2}{|l|}{$\begin{array}{l}\text { Average misclosure } \\
\text { per observation time }\end{array}$} & \multicolumn{2}{l|}{$\begin{array}{l}\text { Computed Relative } \\
\text { Errors of misclosure }\end{array}$} \\
\cline { 2 - 5 } & $\begin{array}{l}\text { Horizontal } \\
(\mathrm{m})\end{array}$ & $\begin{array}{l}\text { Vertical } \\
(\mathrm{m})\end{array}$ & $\begin{array}{l}\text { Horizontal } \\
(\mathrm{m})\end{array}$ & $\begin{array}{l}\text { Vertical } \\
(\mathrm{m})\end{array}$ \\
\hline $2 \mathrm{~min}$ & 0.018 & 0.013 & $1: 1300000$ & $1: 1800000$ \\
\hline $5 \mathrm{~min}$ & 0.017 & 0.010 & $1: 1400000$ & $1: 2500000$ \\
\hline $10 \mathrm{~min}$ & 0.016 & 0.009 & $1: 1400000$ & $1: 2600000$ \\
\hline $15 \mathrm{~min}$ & 0.013 & 0.017 & $1: 1700000$ & $1: 1400000$ \\
\hline $30 \mathrm{~min}$ & 0.011 & 0.030 & $1: 2200000$ & $1: 800000$ \\
\hline 1 hour & 0.010 & 0.009 & $1: 2300000$ & $1: 2600000$ \\
\hline 2 hours & 0.007 & 0.010 & $1: 3400000$ & $1: 2500000$ \\
\hline
\end{tabular}

Table 6. Average misclosures and relative precisions of GNSS observations for all durations.

\begin{tabular}{lcc}
\hline GPS Order & $\begin{array}{l}\text { Traditional Surveys } \\
\text { Order and Class }\end{array}$ & $\begin{array}{c}\text { Relative Accuracy } \\
\text { between Points }\end{array}$ \\
\hline Order AA & & $1: 100,000,000$ \\
Order A & & $1: 10,000,000$ \\
Order B & First Order & $1: 1,000,000$ \\
Order C-1 & Second Order & $1: 100,000$ \\
& Class I & $1: 50,000$ \\
Order C-2-I & Class II & $1: 20,000$ \\
Order C-2-II & Third Order & \\
& Class I & $1: 10,000$ \\
Order C-3 & Class II & $1: 5,000$ \\
& & \\
\hline
\end{tabular}

Figure 7. FGCC horizontal control survey accuracy standards of 1984.

\begin{tabular}{lc}
\hline Order and Class & Relative Accuracy Required between Benchmarks \\
\hline First Order & \\
$\quad$ Class I & $0.5 \mathrm{~mm} \times \sqrt{K}$ \\
Class II & $0.7 \mathrm{~mm} \times \sqrt{K}$ \\
Second Order & $1.0 \mathrm{~mm} \times \sqrt{K}$ \\
Class I & $1.3 \mathrm{~mm} \times \sqrt{K}$ \\
Class II & $2.0 \mathrm{~mm} \times \sqrt{K}$ \\
Third Order &
\end{tabular}

Note: $K$ is distance between benchmarks in kilometers.

Figure 8. FGCC vertical control survey accuracy standards of 1984.

\subsection{Root Mean Square Error}

GNSS accuracy and precision measures are typically given as Circular Error Probable (CEP), RMS, twice the distance of the RMS (2DRMS), and/or radius 95\% (R95) (Ghilani, 2010). For this study, RMS (68.3\% probability level) will be used as a measure of accuracy. RMSE shows variability of the measurements against an accepted value and the equations used for this study are shown in Eqs. 1 and 2 (Ogaja, 2011). Figures 9 to 16 below shows the RMSE's in the horizontal and vertical of the GNSS observations at every observation durations using different satellite combinations.

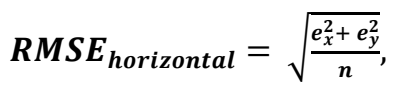

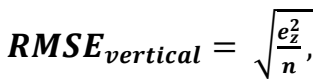

where $e_{x}, e_{y}, e_{z}=$ residuals in position and elevation

$$
n=\text { number of residuals }
$$

As mentioned in Section 3.1, the biases in the MMA3289 observations will be removed in the analysis of the rapid static observations and in the discussions below because the magnitude of the deviations in the horizontal and elevation observations affects the computations and visualization of the results. For example, Figure 9 shows stark difference against Figure 10. Figure 9 shows the RMSE of the horizontal and vertical observations without removing the biases in position and elevation while Figure 10 shows the RMSE in the horizontal and vertical observations with the biases removed. Figure 9 would imply that the accuracy of the vertical observations for the various rapid static durations is between four to five times less than the accuracy of the horizontal observations when in fact the accuracy of GNSS observations for the vertical closely follows the accuracy of the horizontal observations (Figure 10). Figure 9 also implies that the RMSEs of both the horizontal and vertical observations for all durations are almost constant (i.e. horizontal line) around certain values (around $10 \mathrm{~cm}$ for the position and around $50 \mathrm{~cm}$ for the vertical observations). These observations show the importance of checking on a regular basis the consistency of ground control points used as reference stations relative to other control points. If the deviations in the position and elevation of MMA3289 were left unchecked, it would have introduced systematic errors to future measurements whenever it shall be used as a reference control point.

GNSS RMSE

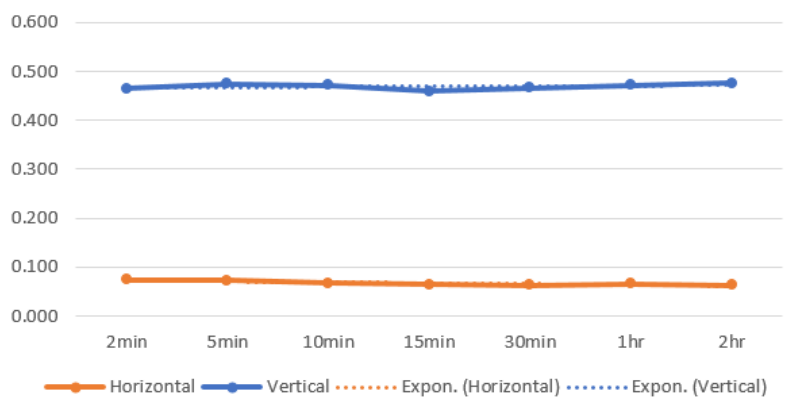

Figure 9. RMSE of horizontal and vertical observations using all GNSS signals with the biases in horizontal and vertical observations in MMA3289 still retained. Y-axis in meters. 
GNSS RMSE (bias removed from MMA3289)

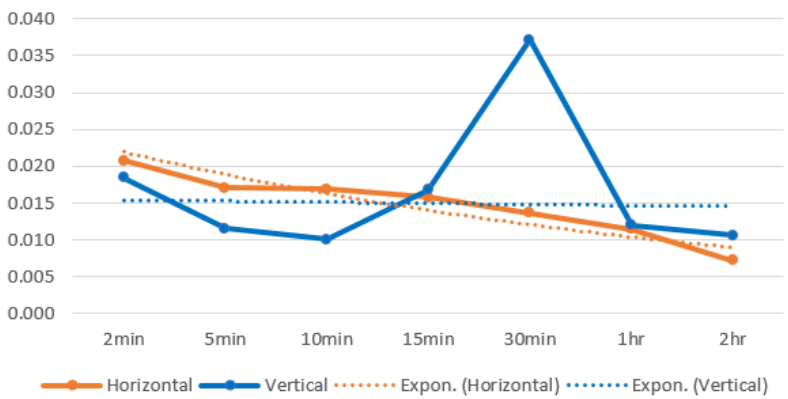

Figure 10. RMSE of horizontal and vertical observations using all GNSS signals. Y-axis in meters.

GPS RMSE

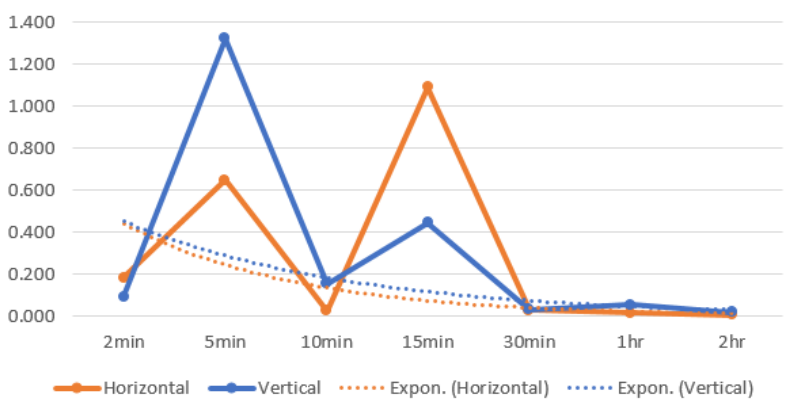

Figure 11. RMSE of horizontal and vertical observations using GPS only. Y-axis in meters.

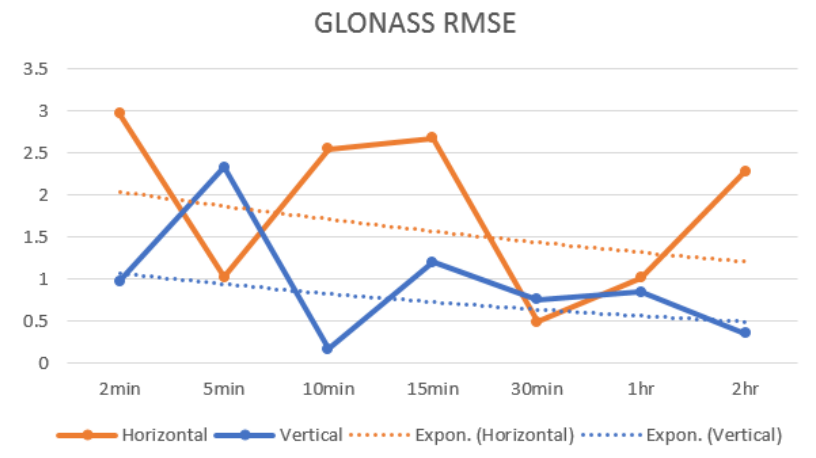

Figure 12. RMSE of horizontal and vertical observations using GLONASS only. Y-axis in meters.
Beidou RMSE

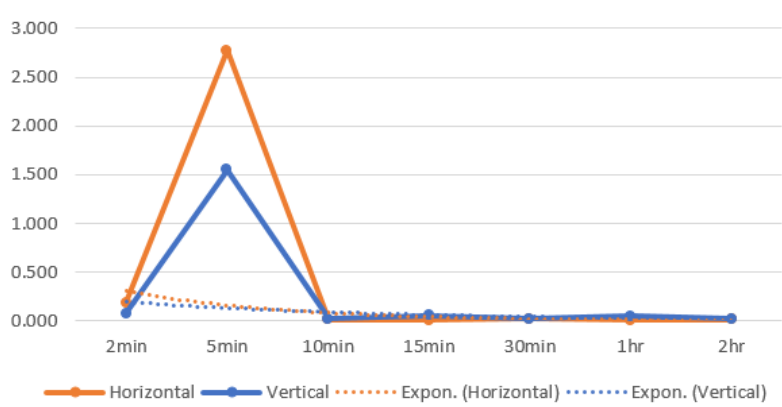

Figure 13. RMSE of horizontal and vertical observations using BeiDou only. Y-axis in meters.

GPS and Beidou RMSE

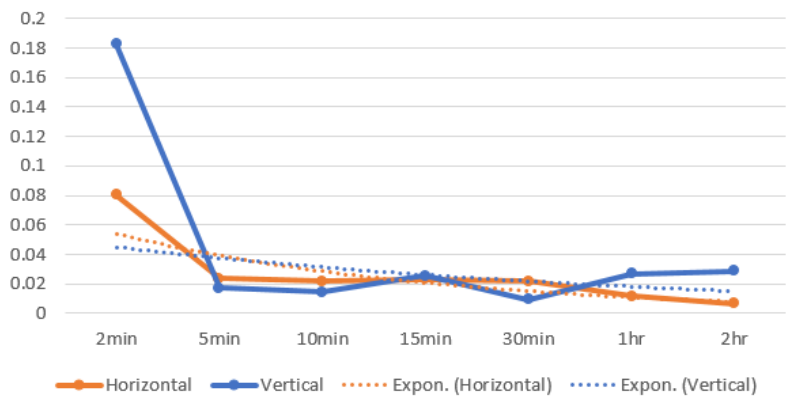

Figure 14. RMSE of horizontal and vertical observations using GPS and BeiDou satellites. Y-axis in meters.

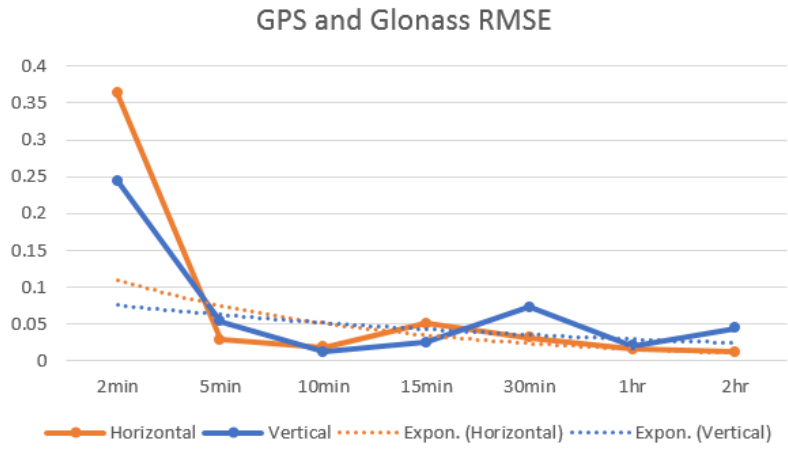

Figure 15. RMSE of horizontal and vertical observations using GPS and Glonass satellites. Y-axis in meters 


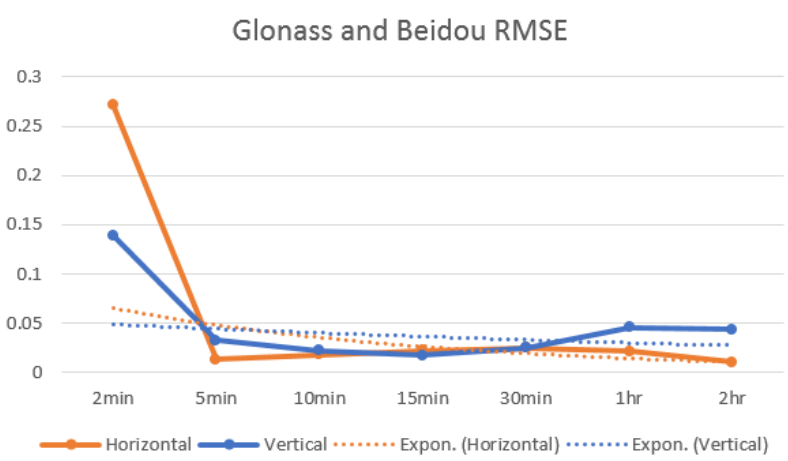

Figure 16. RMSE of horizontal and vertical observations using Glonass and Beidou satellites. Y-axis in meters

As seen in Figure 10, RMSE for both the horizontal and vertical observations do not fluctuate significantly (i.e. lies between 5 $20 \mathrm{~mm}$, except for the outlier in the vertical for the 30-min observation) for the 2-, 5-, 10-, 15-, and 1- and 2-hr observations if all GNSS signals are used in the computations. Horizontal RMSE also indicates that all observation durations are accurate since the values do not deviate so much from the abscissa (i.e. small RMSE implies accurate readings). This implies that, based from the results of this study, if all satellites (GPS, Glonass, BeiDou, QZSS, and Galileo) and all frequencies (L1, L2, L5, etc.) are used in determining the position, as short as 2 minutes of observations can be used. However, for elevation observations, care must be taken as the computed values may fluctuate.

GPS-only (Figure 11) and BeiDou-only (Figure 13) also behave similar to typical GNSS accuracy plots in a manner that the RMSE's go down towards the x-axis at the 30-60 minute mark (El-Rabbany, 2002). The RMSE for GPS-only can even go down faster to the 10-minute mark if the outlier at the 15minute mark is removed (Figure 11). Note however that the GLONASS-only observations (Figure 12) shows an erratic behaviour at all observation intervals.

Significant improvement in the RMSE plots can be observed when at least two satellite constellations are included in the computation of coordinates. As can be seen in Figs. 14, 15, and 16, horizontal RMSE go down starting the 5-minute mark to around less than $5 \mathrm{~cm}$ (i.e. roughly less than 2") for all satellite combinations (i.e. GPS-BeiDou, GPS-Glonass, and GlonassBeiDou). For the vertical RMSE, maximum of less than $10 \mathrm{~cm}$ is observed for the GPS-Glonass combination. Better RMSE in the vertical is observed in the Glonass-BeiDou and GPSBeiDou combinations. These improvements in RMSE are despite the fluctuations seen in the single-satellite RMSE plots in Figs. 11 to 13. It is deemed that the aforementioned improvements are considerable, given that the fluctuations in the RMSE in the single-satellite observations reach up to around 3 meters in the horizontal (Figs. 12 and 13) and 1.4 meters in the vertical (Fig. 11).

\section{CONCLUSIONS AND RECOMMENDATIONS}

\subsection{Conclusions}

As seen in Section 3, using all GNSS signals in baseline processing and network adjustment gives the best positional accuracy in both the horizontal (i.e. Northings and Eastings) and vertical (i.e. height) observations (Fig. 10).
It can also be said that computed RMSE based from field observations generally conform to the RMSE declared by manufacturers of their GNSS instruments (i.e. within $3 \mathrm{~mm}+$ $0.5 \mathrm{ppm}$ horizontal and $5 \mathrm{~mm}+0.5 \mathrm{ppm}$ vertical) especially for observations that are at least 5 minutes long when using (1) all GNSS signals, (2) those using GPS-only and BeiDou-only signals and (3) observations using at least two satellite signals in combination. Glonass-only solution is not recommended since the resulting RMSE plot show erratic behaviour (Fig. 12).

RMSE for horizontal observations when using all GNSS satellites fall between around $5 \mathrm{~mm}$ to $2 \mathrm{~cm}$ while for the vertical, it is around $1 \mathrm{~cm}$ to $4 \mathrm{~cm}$. Note that this observation conforms to the "rule-of-thumb" that vertical observations have half the precision of horizontal observations.

Significant improvements in RMSE plots are observed when information from at least two satellite constellations are included in the network adjustment of rapid static GNSS observations, as seen in Figs. 14 to 16. Based from the plots, it can be said that the minimum observation time that can be used for engineering surveys in urban environments, at least for the case in Metro Manila, is around the 5-minute mark. It is recommended that for better results, GPS-BeiDou combination be considered first, followed by Glonass-BeiDou, and lastly by GPS-Glonass combination.

In addition to the findings above, positional shift was also detected in both horizontal (average $0.114 \mathrm{~m}$ ) and vertical (about $-0.812 \mathrm{~m}$ ) observations done at the NAMRIA fourthorder control MMA3289. It is recommended that this be relayed to NAMRIA and further investigations be made. Note that the reference stations used in the computations are four GCPs that are registered to NAMRIA as second-order controls.

Given that the COVID-19 pandemic is still prevalent and that it limits the duration Land Surveyors can spend time on the field to conduct GNSS observations (i.e. due to physical distancing protocols and curfews), it was found necessary to evaluate the most efficient rapid static observation duration that can be done without sacrificing (1) accuracy and precision of the engineering survey measurements and (2) the safety and health of the Surveyors. As shown in this research, the tipping point is at around the 5-minute observation mark (Fig. 10 and Figs. 14 to 16$)$.

However, if classical accuracy standards would be the basis, rapid static observations as short as 2 minutes may be used for both the horizontal (i.e. meets Class B standards) and vertical (i.e. mostly meets the Third Order classification) measurements, provided that the Land Surveyor be aware that these are superseded standards already and that fluctuations in RMSE are a possibility as shown in Figs. 10 to 16.

\subsection{Future works}

It is planned that other measures of accuracy (i.e. CEP, R95, etc.) will also be computed in the future. Other GNSS equipment may also be used to see if instrumentation (i.e. different GNSS boards, different phase centers, etc.) influences the accuracy of the measurements. Variation of observations through distances instead of time are also being investigated. Other GNSS observation modes, like radio-RTK and RTK via NTRIP, are also being considered for future studies.

Measurements at actual locations, for example beside power poles near heavy vehicular traffic and/or on valves inside urban 
canyons, are also being considered for future work so that these observations may refine the findings of this study.

\section{REFERENCES}

El-Rabanny, A., 2002: Introduction to GPS: The Global Positioning System. Artech House.

Fan, P., Li, W., Cui, X., Lu, M., 2019. Precise and Robust RTKGNSS Positioning in Urban Environments with Dual-Antenna Configuration. MDPI Sensors, 19(3586). doi:10.3390/s 19163586

Federal Geodetic Control Committee (FGCC), 1984: Standards and Specifications for Geodetic Control Networks. US National Geodetic Survey.

Federal Geodetic Control Committee (FGCC), 1998: Geospatial Positioning Accuracy Standards Part 2: Standards for Geodetic Networks. US National Geodetic Survey.

Ghilani, C. D., 2010: Adjustment Computations: Spatial Data Analysis, $5^{\text {th }}$ edition. Wiley and Sons.

Ghilani, C. D., Wolf, P. R., 2012: Elementary Surveying: An Introduction to Geomatics, $13^{\text {th }}$ ed. Pearson.

Gopi,S., Sathikumar, R., Madhu, N., 2018: Advanced Surveying: Total Station, GPS, GIS and Remote Sensing, $2^{\text {nd }}$ ed. Pearson.

Intergovernmental Committee on Survey and Mapping, 2014: Guideline for Continuously Operating Reference Stations. Commonwealth of Australia.

Land Management Bureau (LMB), 2010: Manual on Land Survey Procedures. Department of Environment and Natural Resources.

Li, T., Zhang, H., Gao, Z., Chen, Q., Niu, X., 2018. HighAccuracy Positioning in Urban Environments Using SingleFrequency Multi-GNSS RTK/MEMSIMU Integration. MDPI Remote Sensing. 10(205); doi:10.3390/rs10020205.

National Geodetic Survey, 2020: OPUS: Online Positioning User Service. https://geodesy.noaa.gov/OPUS/about.jsp (11 September 2021).

Ogaja, C. A., 2011: Geomatics Engineering: A Practical Guide to Project Design, CRC Press

Royal Institute of Chartered Surveyors, 2020: Beyond COVID19: Valuation approaches and evidence during the COVID-19 health crisis. RICS.

Van Sickle, J., 2001: GPS for Land Surveyors, $2^{\text {nd }}$ ed. Taylor and Francis. 